\title{
Integrating Function-Directed Treatments into Palliative Care
}

\author{
Andrea L. Cheville, MD, MSCE, Melissa Morrow, PhD, Sean Robinson Smith, MD, \\ Jeffrey R. Basford, MD, PhD
}

\begin{abstract}
The growing acceptance of palliative care has created opportunities to increase the use of rehabilitation services among populations with advanced disease, particularly those with cancer. Broader delivery has been impeded by the lack of a shared definition for palliative rehabilitation and a mismatch between patient needs and established rehabilitation service delivery models. We propose the definition that, in the advanced cancer population, palliative rehabilitation is function-directed care delivered in partnership with other clinical disciplines and aligned with the values of patients who have serious and often incurable illnesses in contexts marked by intense and dynamic symptoms, psychological stress, and medical morbidity to realize potentially time-limited goals. Although palliative rehabilitation is most often delivered by inpatient physical medicine and rehabilitation consultation/liaison services and by physical therapists in skilled nursing facilities, outcomes in these settings have received little scrutiny. In contrast, outpatient cancer rehabilitation programs have gained robust evidentiary support attesting to their benefits across diverse settings. Advancing palliative rehabilitation will require attention to historical barriers to the uptake of cancer rehabilitation services, which include the following: patient and referring physicians' expectation that effective cancer treatment will reverse disablement; breakdown of linear models of disablement due to presence of concurrent symptoms and psychological distress; tension between reflexive palliation and impairment-directed treatment; palliative clinicians' limited familiarity with manual interventions and rehabilitation services; and challenges in identifying receptive patients with the capacity to benefit from rehabilitation services. The effort to address these admittedly complex issues is warranted, as consideration of function in efforts to control symptoms and mood is vital to optimize patients' autonomy and quality of life. In addition, manual rehabilitation modalities are effective and drug sparing in the alleviation of adverse symptoms but are markedly underused. Realizing the potential synergism of integrating rehabilitation services in palliative care will require intensification of interdisciplinary dialogue.
\end{abstract}

\section{Introduction}

It is ironic that although the maintenance of functional independence is central to the quality of life (QoL) of patients with cancer, its loss remains poorly recognized and undertreated $[1,2]$. This disjuncture arises from a number of causes, including the fact that physicians and patients alike often do not discuss its occurrence in their conversations, see treatment of the cancer as the most effective way to address its presence, and may view its progression as an inevitable consequence of cancer. This situation is particularly unfortunate, because effective, established, and often relatively simple rehabilitation treatments are widely available. An additional complication is that rehabilitation services tend to be more effective in the early stages of cancer-related functional loss, a time when patients and clinicians are focused on treatment of the malignancy and not the remediation of its functional effects.

The picture is, in some ways, better in the later stages of disease, when losses are frequently obvious and often devastating. Oncologic clinicians and most patients support conventional rehabilitation in hospitals (eg, consultation liaison services), as well as in post-acute care settings such as inpatient rehabilitation facilities and skilled nursing facilities. Even here, the picture is mixed, as rehabilitation clinicians faced with patients with far advanced disease may question the appropriateness providing intensive and costly services at the terminal stages of a disease [3]. 
The recent ascendance of palliative care, fueled by evidence that its provision improves care and lowers costs [4], has created a climate that may provide an opportunity for the broader integration of rehabilitation services into the continuum of cancer care. In particular, Temel et al's 2010 report that the provision of palliative care to patients with newly diagnosed stage IV lung cancer not only increased survival but also was accompanied by improvements in their QoL and other important clinical outcomes [5] triggered a radical attitudinal shift. In fact, in 2016, the American Society of Clinical Oncology published a recommendation that all patients with advanced cancer be referred for interdisciplinary palliative care [6]. Although subsequent work has not replicated palliative care's survival benefit, it has reinforced its ability to improve QoL and to reduce health care use [7-9].

As yet, the growing acceptance of palliative care has not been associated with a documented commensurate increase in the use of rehabilitation services. Regrettably, functional maintenance and rehabilitation receive only cursory mention in palliative care fellowship curricula and textbooks. It is telling that less than $2 \%$ of the content the Hospice and Palliative Medicine Board Examination relates to rehabilitation service provision [10]. The result is that a majority of palliative medicine practitioners have had, at best, limited exposure to rehabilitative interventions and minimal training in when or how to request them.

Fortunately, recognition of the importance of maintaining functional independence has reached the point where it is spurring widespread efforts to highlight the need for evidence-based cancer and palliative rehabilitation [11,12]. This article targets the latter and is designed to further this goal by looking at palliative care and rehabilitation through the prism of the advanced cancer population and doing the following: (1) proposing a definition of "palliative rehabilitation"; (2) reviewing palliative rehabilitation care delivery models; (3) providing an overview of the barriers that sustain an underuse of rehabilitation services; and (4) describing the strengths and weaknesses of our current rehabilitation interventions, as well as strategies to adapt them to palliative contexts.

\section{Definition of Palliative Rehabilitation}

Although it may seem pedantic, a definition of what palliative rehabilitation entails is necessary to facilitate discourse regarding its clinical and research applications. This exercise is necessary, in part, because the field has redefined itself over the almost 50 years that have passed since Dietz distinguished "palliative rehabilitation" from restorative, supportive, and preventive rehabilitation, as function-directed care delivered to patients with cancer in the far-advanced stages of their illness. Effectively, patient characteristics-prognosis and extent of disease-were Dietz's proposed basis for distinguishing "palliative" from other forms of rehabilitative care. In recent years, palliative medicine has extended increasingly into earlier stages of illness, with some advocating that it be introduced at initial diagnosis. This reconceptualization has expanded the populations, contexts, and goals of palliative care such that in its broadest view, palliative care is essentially QoL-directed supportive care delivered at any point along the trajectory of a progressive illness. This report is focused on patients with advanced, and usually incurable, oncological disease.

The peer-reviewed literature offers some indication of the clinical situations in which the delivery of rehabilitation services may benefit patients with advanced disease and/or intense or refractory symptoms. Specifically, conventional conditioning and resistive exercise can improve physical function and, in some contexts, fatigue. However, whether general conditioning activities for high-performing patients with stage III and IV cancer, for example, Eastern Cooperative Oncology Group (ECOG) 0-1 patients who are experiencing little to no manifestation or associated disablement, should be considered "palliative rehabilitation" is questionable. On the other hand, to confine "palliative rehabilitation" to function-directed services delivered to patients who are imminently dying is to short-change its potential.

Between these ends of the spectrum, uncertainty persists as to where the boundaries that define and distinguish palliative rehabilitation should be drawn. In essence, there is no consensus as to whether palliative rehabilitation should be defined by: (1) the characteristics of the patients it treats, for example, prognosis, cancer stage, level of disablement; (2) the training and skill set of its practitioners; (3) the settings where it is practiced, for example, a hospice; (4) the intense or refractory nature of its therapeutic targets; (5) the frequently limited and transient nature of its goals; or (6) the therapeutic modalities and types of rehabilitation service that are used. In summary, the goal of clearly defining the nature of palliative rehabilitation is not a trivial taxonomic exercise. The lack of a definition results in poorly framed clinical discourse, unfocused goals, and a lack of shared understanding that impairs both care and research.

Examining what palliative rehabilitation is not may help to establish its scope and to highlight its unique dimensions. Palliative rehabilitation, for example, is rarely predicated on conventional models of impairment-driven disablement (eg, the Nagi Model in Figure 1), which implies that disablement and handicap are the end-products of one or a limited number of discrete impairments. Although these models do a good job of explaining the downstream functional consequences of "single hit" focal traumatic, ischemic, and musculoskeletal injuries, they are simplistic and poorly 


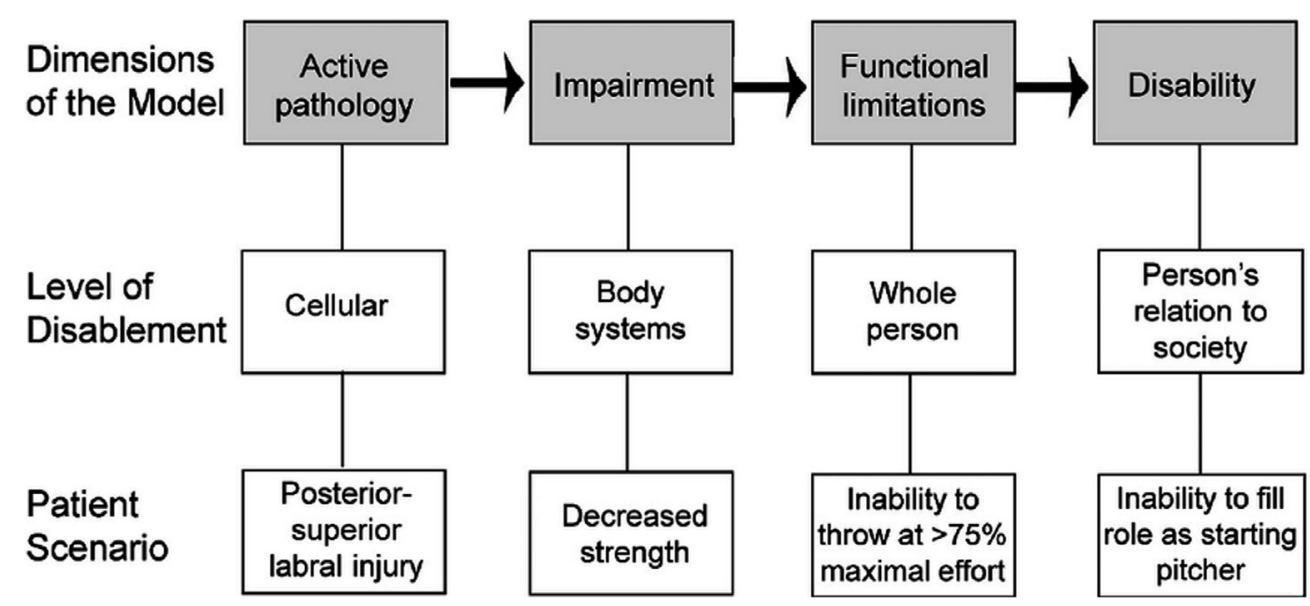

Figure 1. The Nagi Model of Disability.

emphasizes the interplay of factors that drive disablement in patients with chronic, progressive, and concurrent morbidities for whom intense symptoms, existential distress, psychological morbidity, and frailty all collude to degrade function.

It is also useful to consider the results of needs assessments conducted among multi-morbid patients with progressive illnesses [13-15]. These inventories identify that most patients prioritize a need for help in understanding their condition's anticipated impact on function, and other QoL domains. Most express a preference to remain autonomous to the extent that their disease permits, and to protect their lay caregivers from burnout, bankruptcy, and harm [16]. Patients desire symptom control and psycho-emotional support. In addition, a particularly salient desire is for providers to consider patients' highly unique preferences and values. Although this latter is a priority in all rehabilitation service delivery, the staggering range of individual situations and priorities that mark palliative care in patients with advanced cancer is a distinguishing feature.

In addition, a consideration of the skills and orientation required for physiatrists to effectively meet the needs of these patients offers some insight into the nature of palliative rehabilitation. Palliative physiatrists must be able to deliver care in alignment with patient values to comprehensively address threats to patients' waning independence with an emphasis on the following: (1) optimizing symptoms and mood; (2) consideration of existential issues and motivation; (3) the particulars of disease and treatment prognoses; and (4) integration of diverse systems of care and caregivers. Perhaps most importantly, palliative physiatrists must be comfortable with limited and temporary rehabilitation goals and intensely dynamic clinical situations in which many critical factors may change rapidly. In summary, it may be the shift in focus and intention, as well as an intensity of collaboration with other disciplines, that most distinguishes palliative from other forms of rehabilitation in the advanced cancer population. The following working definition is offered as a starting point for discourse:

Palliative rehabilitation is function-directed care delivered in partnership with other disciplines and aligned with the values of patients who have serious and often incurable illnesses in contexts marked by intense and dynamic symptoms, psychological stress, and medical morbidity, to realize potentially timelimited goals.

\section{Delivery Models of Cancer Rehabilitation}

Tension between the potential benefits of palliative rehabilitation and the clinical infrastructure and delivery models that currently direct its administration leads to a mismatch between patients' needs and the services available to them. Patients negotiating intense symptoms, debilitating oncologic treatments, and existential distress may have limited time, resources, and energy to invest in the rehabilitation process. They are frequently unable to repeatedly travel to outpatient clinics and facilities where nonresidential rehabilitation services are conventionally delivered, and identify travel requirements as a barrier [17]. Efforts to leverage technology in the interest of developing less time- and travel-intensive approaches have gained some traction. However, reimbursement beyond standard rehabilitation care delivery settings remains a challenge. Clinical contexts in which palliative rehabilitation may be delivered and the empirical basis for its effectiveness in each are described in this section. It should be noted that the citing of reports is not intended to imply that the described interventions are integral to palliative rehabilitation but, rather, to offer the reader a general overview of the evidentiary basis for providing rehabilitation services to palliative populations. 


\section{Inpatient Facility-Based Palliative Rehabilitation}

\section{Skilled Nursing Facilities}

Skilled nursing facilities (SNFs) are among the most common settings for the delivery of palliative rehabilitation; however, to our knowledge, no reports have described its effectiveness in this context. Because rehabilitation service intensity and goals are relatively less ambitious in SNF-level rehabilitation, it is theoretically a good match for patients with palliative needs.

\section{Inpatient Rehabilitation Facilities}

Patients admitted to inpatient rehabilitation facilities (IRFs) must have sufficient stamina to participate in 3 hours per day of therapy and a high likelihood of being dismissed to home without the requirement for additional residential rehabilitation. This latter presents a challenge for palliative patients who are vulnerable to unexpected interruptions in their rehabilitation therapies $[18,19]$. Evidence for the effectiveness of IRF-based palliative rehabilitation is elusive. Although reports describe comparable Functional Independent Measure (FIM) efficiencies among patients with malignant, relative to traumatic and ischemic, brain and spinal cord injuries, the affected patients are often individuals who would not be conventionally characterized as "palliative," as many continue to aggressively pursue diseasedirected therapies. To our knowledge, there are no reports of controlled comparison of outcomes among classically palliative patients who do and do not receive IRF-based rehabilitation.

\section{Acute Hospital-Based Palliative Rehabilitation}

Services provided by physical medicine and rehabilitation (PMER) hospital-based consultation/liaison services may be the most common and widespread mode of delivering palliative rehabilitation. Such services are delivered by highly specialized groups in comprehensive cancer centers, as well as by PM\&R generalists in community settings. No comparisons have been reported of palliative patients who do and do not receive consultative PM\&R or physical therapy (PT) services. However, reports document improvements in pre to post comparisons of patients' function after service delivery [20].

\section{Residential Hospice- and Palliative Care Ward-Based Palliative Rehabilitation}

Several case series described significant improvement in pre to post PT comparisons among hospice patients [21-23]. More recently, a randomized controlled trial demonstrated benefits in symptom reduction, including fatigue, among hospice residents randomized to PT versus usual care [24].

\section{Outpatient Facility-Based Palliative Rehabilitation}

The evidence supporting facility-based rehabilitation to enhance function and other dimensions of QoL, as well as objective performance measures, is fairly robust, although marked by heterogeneity of samples, settings, and interventions. This variability, in part, explains why findings have consistently differed across reports with respect to the magnitude of effect sizes (ES) in different domains. For example, some studies have detected significant improvements in fatigue, whereas others have not $[25,26]$. Intervention settings have differed substantially; including conventional outpatient rehabilitation settings, cancer centers, recreational facilities, and day hospices. Study samples have also differed with respect to inclusion criteria regarding prognosis and cancer stage. For example, more than one-third of patients described in a case series of center-based, posttreatment, multimodal rehabilitation had stage III cancer, which, although technically "late stage," may be relatively benign [27]. In contrast, other studies have enrolled only patients with life expectancies of 12 months or less [28]. The diversity of intervention components is also striking. Several reports describe multimodal interventions that have combined exercise with nutritional or psychospiritually directed components $[29,30]$. In contrast, other studies have assessed interventions comprising exercise alone [31,32]. Multi-modal interventions, in general, have more consistently improved patientreported outcome (PRO) domains, although their diversity defies broad generalizations.

Overall, however, it becomes clear that physical exercise and multimodal rehabilitation programs have the potential to positively affect a range of clinical outcomes among patients with late-stage cancer. Moreover, reports suggest that interventions may be not only effective but may fall within conventional willingness-to-pay thresholds [33,34].

\section{Hybrid and Home-Based Palliative Rehabilitation}

Several reports attest to the benefits of multimodal home palliative care; however, most do not explicitly clarify the role of rehabilitation services $[35,36]$. Hybrid programs limit travel requirements and may include 1 or 2 clinic sessions or the initiation of hospital-based therapy followed by provision of intensive inter- or postsession home support and instructional materials [37]. A randomized trial of such a hybrid program delivered to patients with stage IV lung and colon cancers noted significant intergroup differences in fatigue and mobility, favoring the intervention group [38]. Unfortunately, some palliative patients are unable to negotiate even streamlined travel requirements. Telecare delivery models that 
expand reach, eliminate travel requirements, and neutralize geographic barriers have been validated in the treatment of pain and depression [39]. A recent effort to explore whether these approaches could be leveraged to deliver rehabilitative services was promising. More specifically, a 42-patient pilot study of a fatigue-directed intervention leveraged extensive prior work and assessed the benefits of administering a behavioral intervention that integrated resistive and aerobic exercise with mindfulness-based cognitive rehabilitation over 8 one-toone telephonic sessions to patients with late-stage cancer [40-42]. This pilot work established that the intervention had many desirable attributes: (1) multidomain effectiveness: the pre-post pilot ESs were 0.48 for fatigue interference, 0.51 for QoL, and 0.42 for physical function; (2) high acceptability: $83 \%$ of participants $(\mathrm{N}=35)$ requested continued support after completing the pilot study; and (3) tolerability: all participants were able to perform the recommended behaviors. In accordance with reports in the literature, less than $15 \%$ of pilot participants preferred in-person to telecare [43-45]. This work suggests that teledelivery may be an effective, cost-sensitive, and patient-centric means of delivering palliative rehabilitation services to patients with advanced cancer.

\section{Barriers to the Uptake of Palliative Rehabilitation}

The reasons for the underuse of rehabilitation services in the advanced cancer population are multifactorial, and both oncologic and rehabilitation clinicians likely affect referral patterns. Oncologists' attitudes toward rehabilitation services have been characterized only to a limited extent $[3,46]$. A mixed picture emerges. On one hand, it is challenging for busy oncological clinicians to single out issues for referral other than for discrete and problematic clinical issues that fall outside of their scope of expertise, such as lymphedema [47]. However, on average, patients with late-stage cancer have multiple physical impairments and function-degrading symptoms for which no referrals are made. Often when patients concurrently experience pain, depression, or anxiety along with functional loss, they may be referred to specialists in disciplines such as pain management and psychiatry, rather than rehabilitation.

\section{Patient and Referring Physicians' Expectation That Effective Cancer Treatment Will Reverse Disablement}

Referral patterns and logistical barriers are only part of the problem. Qualitative work on patients' receptivity to rehabilitation services may shed light on referring practices as well. Cheville et al have shown that when patients with stage III and IV lung cancer who were experiencing moderate or severe $(\geq 4 / 10$ on an 11-point numerical rating scale) functional losses and levels of associated distress were asked if they were interested in receiving rehabilitation services, more than two-thirds were not [17]. The reasons for their lack of receptivity included a desire to wait until the effects of their cancer treatment or findings of their next scheduled imaging studies were known, as well as general skepticism about the benefits of rehabilitation. It was also apparent that they assumed that since cancer was the cause of their functional losses, that cancer treatment was the most effective means of regaining function. This belief, unfortunately, is contrary to the fact that cancer-related disablement is progressive and persists or advances insidiously regardless of treatment

Indeed, unrealistic expectations and concerns shared by oncologists and other clinicians caring for patients with advanced cancer have proved to be barriers to rehabilitation referrals. Runacres et al found that although oncologists viewed rehabilitation for cancer patients favorably, and understood that palliative care physicians did not necessarily have the requisite rehabilitation skillset to provide medical rehabilitation, many oncologists were hesitant to refer advanced cancer patients for rehabilitation services out of concern that it would inspire false hope in patients about their overall prognosis [48]. Patients are often more optimistic about their prognosis than oncologists, and their caregivers often believe that oncologists do not understand the extent to which patients with advanced cancer can recover from physical and cognitive deficits $[49,50]$. An understanding of these perspectives is essential to providing excellent palliative care, and is perhaps why oncologists more comfortable with end-oflife issues are more likely to refer to palliative care [51].

The idea that effective mitigation of a primary disease process will automatically reverse its sequelae is implicit in the biomedical model and remains so despite growing evidence to the contrary [52,53]. The introduction of the use of novel biologicals has resulted in the expansion of survival times, but in turn may be associated with toxicities exceeding those reported in the initial clinical trials [54]. There is a critical need for patient engagement by aligning the benefits of rehabilitation services with patients' personal goals, and for patient and clinician education regarding the need for rehabilitation services, given the progressive nature of cancer-related disablement despite effective anticancer treatment.

\section{Breakdown of Linear Model of Disablement Due to Presence of Concurrent Symptoms and Psychological Distress}

Functional loss in the palliative setting is often complex. Although the International Classification of Function and Disablement (ICF) (Figure 2) acknowledges greater complexity and more contributing factors than its earlier Nagi forerunner (Figure 1), an implicit linearity is still present. Both models suggest that a discrete or a limited number of impairments produce disability that, 


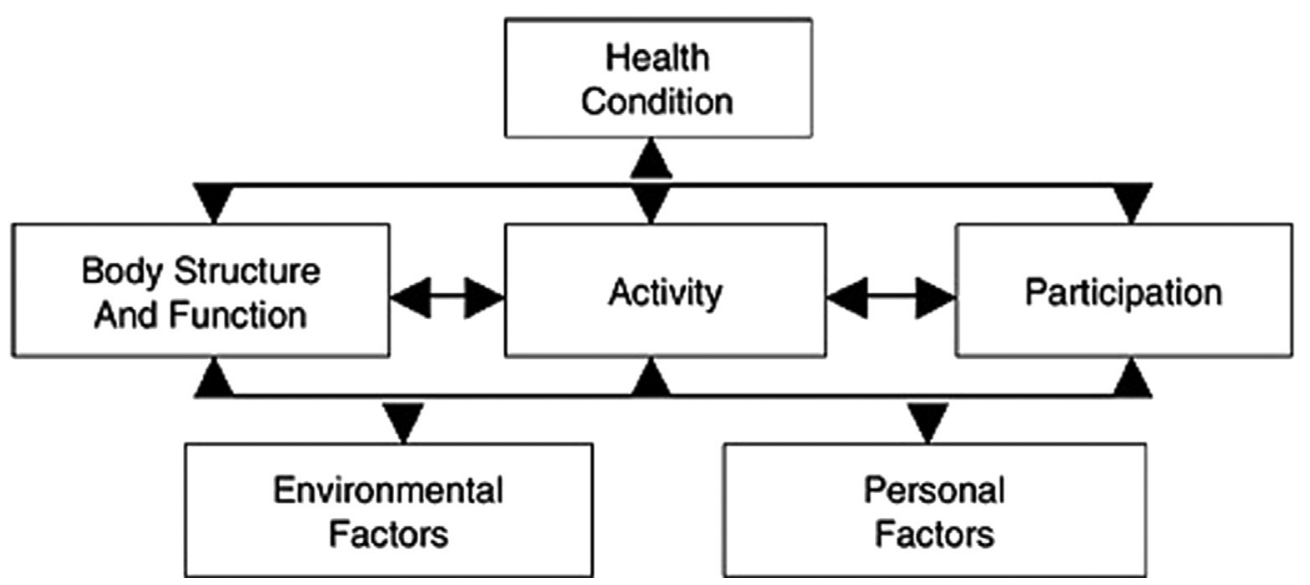

Figure 2. International Classification of Functioning, Disability, and Health.

contingent on demographic and social factors, produces contextually specific handicaps. Although these relatively obvious relationships do occur in the palliative setting - such as with malignant spinal cord compression or a hemorrhagic brain metastasis-they are not the norm. Rather than isolated, functionally devastating impairments, patients more often accrue multiple impairments, with the average patient in the late stages of cancer having upward of 5 [2]. Furthermore, work by several groups has confirmed that pain and fatigue are principal determinants of disablement in patients with late-stage cancer [53]. Given this, rehabilitation efforts that fail to adequately address clinical domains beyond physical impairments are unlikely to succeed. Nevertheless, current models of rehabilitation service delivery consider the relevance of these domains only to a limited extent, for example, mood and "motivation."

Fortunately, our ability to detect and manage these domains in chronically ill populations has advanced dramatically. One of the most important changes has been the validation and clinical integration of PROs linked to treatment recommendations. The Patient Health Questionnaire-9 (PHQ-9) is perhaps the most salient example. The PHQ-9 was developed for depression screening in primary care settings; however, it has now been validated in diverse clinical populations, including patients with advanced cancer [55]. Scores have been linked to levels of clinical acuity and management recommendations. The benefits of this extensive work have been leveraged to a limited extent in rehabilitation settings, even though depression is prevalent among disabled cancer populations. In the palliative setting, a failure to screen for depression with the PHQ-9 or an alternative PRO may severely undermine outcomes. Management can be directed by guidelines or per clinician discretion. The 7-item Generalized Anxiety Disorder scale (GAD-7), similar to the PHQ-9, is a widely used screening tool, in this case for anxiety [56], which is also highly prevalent among palliative populations.
Similar to the efforts that have linked PHQ-9 score intervals with specific treatment recommendations, work using the Brief Pain Inventory [57] and the Patient Reported Outcome Measurement Information System (PROMIS) has identified score ranges associated with mild, moderate, and severe levels of symptom intensity, with well established guidelines available to inform the management of symptom intensities across this continuum. In addition to pain and fatigue, in advanced cancer dyspnea, nausea, degraded sleep quality, and cough are prevalent symptoms that may also be screened for and graded using PROs. This approach permits the quantification of a patient's symptoms and allows tracking over time to assess progression and treatment response.

Existential distress inevitably attends progressive disablement, especially in the setting of a terminal illness, with patients often being forced to relinquish their defining familial, social, and vocational roles as their illnesses progress. In the rehabilitation settings, this process may be further hampered by clinicians' limited familiarity or comfort with more humble goals than lasting community and vocational reintegration. Impactful work conducted by Chochinov et al [58] offers clinicians and patients a way forward in their efforts to redefine meaning and to identify and foster the essential elements of personhood and dignity in advanced illness.

\section{Tension Between Reflexive Palliation and Impairment/Disease-Directed Treatment}

Although accurate diagnosis is integral to the practice of palliative medicine, it is often superseded by the need to acutely relieve symptoms and distress. Frequently the relative importance of the contributors to a patient's distress cannot be definitely identified in the time available, or the required diagnostic efforts are untenable or unjustified in the context of far-advanced illness. In this manner, workup may exact unacceptable opportunity costs that delay or displace efforts to optimize comfort. However, reflexive pharmaceutical palliation 
without consideration of reversible and locally treatable causes is seldom acceptable and may produce needless side effects. Physiatrists can bring refined neuromuscular diagnostic skills that are not routinely available in the palliative setting and may identify previously unrecognized drivers of pain and impairment. However, an inappropriate focus on neuromuscular diagnostics can be counterproductive, and a tension may arise between the desire to practice impairment/diagnosis-driven rehabilitation and the need to acutely palliate despite diagnostic uncertainty. Ideally this tension can be diffused when the expertise of palliative clinicians to systemically alleviate suffering is paired with the neuromuscular diagnostic precision of physiatry.

To realize potential synergism, physiatrists must recognize the gaps in clinical care that their knowledge and skills may bridge, and concurrently develop comfort with the high-dose, aggressive systemic palliation that may be required to relieve suffering. The latter may need to be addressed, particularly in the late stages of disease, with the use of opioids and other pharmacological agents that are controlled, potentially addictive, and centrally acting.

\section{Palliative Clinicians' Limited Familiarity With Manual Interventions and Rehabilitation Services}

The palliative care work force is changing under the influence of multiple factors that powerfully influence rehabilitation service delivery. Specifically, the rate of credentialing of new trainees is generally acknowledged to be insufficient to meet the needs of a rapidly expanding aged, frail, and multimorbid patient population. This has resulted in an increasing reliance on mid-level providers to populate palliative care teams, predominantly nurse practitioners, physician assistants, and clinical nurse specialists, few of whom have worked in settings where the detection and mitigation of disablement was an important factor. To a lesser degree, the same lack of familiarity applies to palliative care physicians, the significant majority of whom subspecialize in palliative care from general internal medicine, anesthesiology, family medicine, or psychiatry, disciplines that variably emphasize functional preservation in their scope of practice.

Currently, only $1 \%$ of the physicians certified in Hospice and Palliative Medicine by the American Board of Internal Medicine are physiatrists [59]. It is not known how they are practicing, or the extent to which their activities are integrated into conventional palliative care services. In general, there has been limited systematic integration of rehabilitation services into either inpatient or outpatient palliative care or hospice programs [48]. As a consequence, familiarizing the palliative care work force with the potential contributions of rehabilitation medicine will continue to depend on the efforts of currently practicing physiatrists who may have limited experience in palliative rehabilitation.

\section{Challenges in Identifying Receptive Patients With the Capacity to Benefit From Rehabilitation Services}

Unlike many of the traumatic or ischemic injuries that cause acute and marked declines in function and are the typical purview of rehabilitation medicine, disablement in the palliative setting is insidiously progressive and marked by an acceleration toward the end of life. As previously mentioned, many patients attribute their functional losses to their diseases and are willing to a limited extent to engage in the rehabilitative process until their losses have become pronounced and problematic. Unfortunately, many patients and their treating clinicians wait until patients are hospitalized and disablement has progressed to the point of impeding home dismissal. This approach results in needless institutionalization and becomes even less acceptable in light of spiraling costs and patient numbers. However, 2 elements vital to creating a credible alternative have not yet enjoyed broad clinical uptake. These include systematic screening for functional decline, and proactive effort on the part of oncology care teams to make functional preservation an integral part of comprehensive cancer care. Advances in PRO-based functional assessment offer the potential to create unprecedentedly precise and efficient measures to screen for subtle changes in patient functioning over time. This work continues to advance, as described in the next paragraph, but its impact remains limited.

A brief description of ongoing efforts to enhance PRObased functional assessment is warranted, because these approaches offer a pragmatic means of identifying and risk stratifying patients experiencing disablement, and item response theory-based instruments now permit the rapid assessment of latent traits such as mood and pain, which cannot be objectively assessed. At present, 2 item response theory-modeled item banks that evaluate function have been studied in cancer populations: the Activity Measure for Post-Acute Care and the PROMIS function bank, both of which can be administered with greater efficiency and precision using a computer adaptive testing platform. Of these, the Basic Mobility Activity Measure for Post-Acute Care item bank has been psychometrically characterized among patients with advanced-stage cancer and shown to be valid and responsive [53]. The goal of embedding functional screening in routine oncology and palliative care delivery pathways may become far more feasible in the near term, because the latest versions of many electronic health records include the capability to administer item banks via computer adaptive testing. 
Application of Rehabilitation Interventions and Principles in Palliative Care

Palliative rehabilitation services are most consistently engaged in acute care hospital settings. This reflects the disproportionate expansion of inpatient, relative to outpatient, palliative care, with most teaching hospitals now providing some form of palliative care subspecialty services. Rehabilitation's pragmatic focus on function complements the emphasis on symptom management and goal setting that characterizes palliative care.

An important consideration is that since US hospices are overwhelmingly home based, patients and their caregivers are understandably concerned about negotiating the transition from a hospital setting to home, and the requirements of feeding, toileting, and other daily activities. Such mundane concerns are often overshadowed by concerns regarding whether to continue oncology-directed treatment, how to manage refractory pain, code status, and so forth. Rehabilitation specialists are uniquely equipped to foster the concrete, practical discussions that explore the small essential concerns, such as how and whether to use a gait aid or how to get out of the house, that determine the substance of patients' lives as they grapple with larger issues. What follows is a brief guide for the rehabilitation consultant with suggestions for how to appropriately target issues common among patients in the palliative stages of illness.

\section{Pain Management}

Palliative care clinicians are analgesic experts but may not fully recognize the need to address movementassociated pain that powerfully degrades patients' function and autonomy. Bone metastases are the most prevalent source of cancer-related pain [60]. Inevitably, loading or moving the affected structures provokes pain. Immobility is the easiest way to avoid pain, and most patients' default in the absence of appropriate education on how to use their analgesics strategically to control movement-associated pain. Because many patients have diminished pain at rest, the use of long-acting agents to control their incident, movement-associated pain results in overmedication. Lower doses and improved control can be achieved by anticipating intervals of increased movement and premedicating with an adequate dose of a "normal"- or "immediate"- release opioid or nonsteroidal antiinflammatory drug, more often the former. The dose required can be established through an iterative trialand-error process with the understanding that requirements are dynamic and will change over time. All naturally occurring and semisynthetic opioids, for example, morphine, oxycodone, and hydrocodone, require 30-45 minutes after ingestion before taking effect. In contrast, purely synthetic fentanyl is highly lipophilic, and transmucosal preparations can take effect in 3-5 minutes. Unfortunately, these agents are costly, and the requirement for Transmucosal Immediate Release Fentanyl Risk Evaluation and Mitigation Strategy program certification to prescribe them have restricted access.

Rather than suggesting specific agents or doses, the role of the rehabilitation clinician in managing movement-associated pain is usually 2 -fold. First, there is a need to discuss strategic approaches for managing incident pain with the referring primary or palliative care service, with emphasis on the critical need to synchronize periods of movement with the analgesic's serological peak. Second, it is critical to promote understanding of the strategy across all relevant stakeholders including patients, caregivers, nurses, physical and occupational therapists, as well as the relevant medical services. Educating both the patient and the family is essential, given that they will be required to execute the strategy after hospital discharge. Perhaps most importantly, the patient should be afforded the freedom to experiment, because pain distribution, intensity, and provoking movements will inevitably change over time.

\section{Positioning and De-weighting to Control Pain}

Gait aids, positioning, foam bolsters, seat cushions, mattress overlays, and the many other devices used to remove pressure from painful structures are underused in general palliative care. Cachexia is common among patients with advanced disease, and pressure on bony prominences can provoke pain that may prove refractory to even high-dose opioid analgesia. Simple de-weighting techniques, including regular repositioning, offer an inexpensive and often remarkably effective means of addressing focal, pressure-related pain. Approaches are inevitably individual, and generalized prescriptive recommendations are of limited value. More importantly, rehabilitative clinicians should remember to check for opportunities to apply their expertise in seating, offloading, and de-weighting, even when they have not been consulted for this purpose.

\section{Orthotics}

Orthotics are similarly underused in the palliative setting, with the exception of clamshell-type thoracolumbosacral orthoses, which are often reflexively ordered to control back pain. Several overarching principles should guide palliative orthotic prescription. The following recommendations are based on anecdotal experience, as there is little evidence base to direct clinical decision making. First, comfort is paramount, even when a patient's spine may be in future jeopardy. 
Before prescribing an orthotic, which are often expensive, poorly tolerated by patients, and inconsistently covered by third-party payers, effort should be invested in determining whether it will enhance comfort. Difficulty in putting on and taking off, chafing, focal constriction, and so forth are all common issues that undermine the stated treatment goal. The availability of off-the-shelf or loaner models that patients can trial without a costly investment are an asset. Second, patients' future status must be anticipated. Weight loss and gain, edema, progressive weakness, and other issues may render an effective orthotic relatively useless in a short period of time. Patients' anticipated functional abilities and goals may or may not align with the need for an orthotic. Finally, if a patient is dismissing to hospice or home, the requirement for an orthotic and granular prescription details should be explicitly documented.

\section{Edema}

Focal or generalized edema is highly prevalent and is an important source of discomfort and disablement in palliative populations. Contributing factors such as organ failure, hypoalbuminemia, venous obstruction, and lymphatic blockage tend to make the resultant swelling dynamic and refractory. In addition, cardiac and renal failure may impede mobilization and elimination of fluid once returned to the intravascular space. In the palliative context, prudent use of diuretics may be highly beneficial. This contrasts with the conventional management of lymphedema and venous stasis edema, where diuretic use is discouraged. Use of compression garments may be ineffective, given ongoing shifts in fluid volume and distribution, as well as challenges in putting these on. Multilayer short stretch bandaging can generally accommodate such shifts, but is laborious for caregivers and may be untenable for patients with limited assistance. Alternative Velcro-based compression devices such as the Comprefit (BiaCare, Zeeland, MI) and Juxta-fit (mediUSA, Whitsett, NC) adjustable garments often represent the most convenient and effective management option [61]. However, even the most rudimentary of these garments are not inexpensive, and coverage varies across payers.

\section{Activities of Daily Living and Mobility}

Optimizing safety and autonomy during a patient's activities of daily living (ADLs) in the palliative setting differs little from other contexts, with the exception of one important caveat: patients' functional capabilities are dynamically declining, and effective management must anticipate their condition over the weeks to months ahead. This is not a simple task, given the staggering heterogeneity of cancer types and treatments. In addition, many patients are, even when confronting far-advanced illness, hoping to improve, and may not welcome candid discussions of their impending disablement. Clinicians must perform a delicate balancing act of providing patients and their caregivers with the knowledge, skills, and equipment needed to preserve autonomy, without triggering alarm, rejection, or denial.

\section{Hospice and Rehabilitation Services}

Hospice providers may vary in the available hours of home health assistance, respite care policies, patient bathing and toileting, and support in other household maintenance tasks. Rehabilitation clinicians, with their keen awareness of task requirements, can play an invaluable role in assisting patient and their families in the selection of a hospice provider. It should be remembered that, at times, hospice may offer fewer services than traditional home care; therefore, it is essential to consider patient and family needs on a caseby-case basis. Most hospices offer expert management of symptoms, existential distress, anticipatory grief, mood disorders, and other concerns prevalent at the end of life. If such issues are anticipated, hospice may be the best option. However, hospice need not be the reflexive go-to in provision of home services for terminal patients.

Hospice providers are required to comprehensively address patients' needs. However, as mentioned previously, rehabilitation needs may be overshadowed unless the hospital-based rehabilitation team carefully highlights and outlines the patients' equipment, orthotic, and therapy needs as well as encouraging caregivers to advocate to ensure that recommendations are followed. This applies irrespective of whether patients dismiss to home or residential hospice facilities.

Rehabilitation clinicians, with their insights into patient functioning, and caregivers' ability to safely provide assistance can contribute invaluably to decision making in dismissal planning as well as to whether a patient requires residential hospice. Hospice services may be provided in skilled nursing facilities or hospice homes. In either case, unless a patient is covered by Medicaid, has long-term care insurance, or is Veterans' Administration affiliated, out-of-pocket expenses can be substantial. Assisting primary, palliative, and social service workers to sort through conflicting priorities in a hospice dismissal setting is a much appreciated role that rehabilitative providers are uniquely equipped to fulfill.

\section{Recommendations for Physiatrists Providing Palliative Rehabilitation}

Only a limited evidence base is available to guide palliative physiatrists, but the experiences of seasoned 
clinicians provide some insights and are the basis for the following recommendations. First, establish and nurture a supportive relationship. This may involve learning the particulars of a patient's home life as well as the patient's functional goals and expectations. Often patients and their caregivers, fatigued by complex treatment-related discussions, welcome simple and concrete exchanges about the accessibility of their home, getting into and out of their vehicle, and local sources of support. It is important to gain an understanding of how patients and caregivers perceive their current and future functional capabilities. A focus on current abilities and ensuring safety for patients as well as their caregivers may enable patients to maintain dignity while accepting assistance.

Second, gingerly initiate discussion about a patient's potential use of hospice care, or other home services, focusing on who may be with the patient, their level of health and function, and potential pitfalls. Problem solving theoretical barriers may afford enough emotional distance that patients and caregivers are able to engage without feeling threatened or overwhelmed. Describing patients' performance in objective terms, whether they are improving or declining, creates a basis for discussion about dismissal location and the extent to which caregivers may safely manage at home. Encouraging caregivers to actively assist nurses and therapists in mobilizing the patient not only provides vital insight into the difficulties that they may face at home, but offers opportunities to promote safe biomechanics, engage caregivers in equipment selection, and highlight challenging task sequences or components.

Third, proceed cautiously in preparing patients and caregivers for the declines that lie ahead. How far to pursue discussions of future needs is a contextual and highly practitioner-dependent decision. Patients expect to learn their prognoses from their oncologists and are understandably disturbed when information is not delivered consistently across services [62]. It is therefore vital that palliative physiatrists have an up-to-date and accurate understanding of what information has been relayed and by whom regarding prognosis and dismissal planning. If patients and/or caregivers are unable to engage in "what to do in the event of ..." discussions, then developing a plan that includes frequent reassessment and access to rehabilitation services is critical. Often, even if patients are willing and able to have these difficult conversations, all possible contingencies cannot be addressed. Informing patients and caregivers that help is available, and encouraging them have a low threshold to ask for help, is often the best that one can do.

\section{Conclusion}

Palliative rehabilitation is a growing need that remains severely underaddressed. Whether and how
PM\&R rises to the challenge of caring for the expanding population of seriously ill, disabled patients will define the future of the field. The issue is a national priority and should prompt physiatrists and other rehabilitation specialists to question whether the field's scope should be guided by tradition, reimbursement, or patient need.

\section{References}

1. Cheville AL, Kornblith AB, Basford JR. An examination of the causes for the underutilization of rehabilitation services among people with advanced cancer. Am J Phys Med Rehabil 2011;90: S27-S37.

2. Cheville AL, Beck LA, Petersen TL, Marks RS, Gamble GL. The detection and treatment of cancer-related functional problems in an outpatient setting. Support Care Cancer 2009;17:61-67.

3. Spill GR, Hlubocky FJ, Daugherty CK. Oncologists' and physiatrists' attitudes regarding rehabilitation for patients with advanced cancer. PM R 2012;4:96-108.

4. Dalal S, Bruera E. End-of-life care matters: Palliative cancer care results in better care and lower costs. Oncologist 2017;22: 361-368.

5. Temel JS, Greer JA, Muzikansky A, et al. Early palliative care for patients with metastatic non-small-cell lung cancer. N Engl J Med 2010;363:733-742.

6. Ferrell BR, Temel JS, Temin S, et al. Integration of palliative care into standard oncology care: American Society of Clinical Oncology Clinical Practice Guideline update. J Clin Oncol 2017;35: 96-112.

7. Temel JS, Greer JA, El-Jawahri A, et al. Effects of early integrated palliative care in patients with lung and GI cancer: A randomized clinical trial. J Clin Oncol 2017;35:834-841.

8. Bakitas M, Lyons KD, Hegel MT, et al. Effects of a palliative care intervention on clinical outcomes in patients with advanced cancer: the Project ENABLE II randomized controlled trial. JAMA 2009; 302:741-749.

9. Zimmermann C, Swami N, Krzyzanowska M, et al. Early palliative care for patients with advanced cancer: A cluster-randomised controlled trial. Lancet 2014;383:1721-1730.

10. American Board of Internal Medicine. Hospice and Palliative Medicine Certification Examination Blueprint. 2017. Available at http://www. abim.org/ /media/ABIM\%20Public/Files/pdf/exam-blueprints/ certification/hospice-palliative-medicine.pdf. Accessed July 31, 2017.

11. Raj VS, Silver JK, Pugh TM, Fu JB. Palliative care and physiatry in the oncology care spectrum: An opportunity for distinct and collaborative approaches. Phys Med Rehabil Clin N Am 2017;28: 35-47.

12. Gupta AD, Lewis S, Shute R. Patients living with cancer-the role of rehabilitation. Aust Fam Physician 2010;39:844-846.

13. Schenker Y, Park SY, Maciasz R, Arnold RM. Do patients with advanced cancer and unmet palliative care needs have an interest in receiving palliative care services? J Palliat Med 2014;17: 667-672.

14. Ahmed N, Ahmedzai SH, Collins K, Noble B. Holistic assessment of supportive and palliative care needs: The evidence for routine systematic questioning. BMJ Support Palliat Care 2014;4: 238-246.

15. Hwang SS, Chang VT, Cogswell J, et al. Study of unmet needs in symptomatic veterans with advanced cancer: Incidence, independent predictors and unmet needs outcome model. J Pain Symptom Manage 2004;28:421-432.

16. Husain LS, Collins K, Reed M, Wyld L. Choices in cancer treatment: A qualitative study of the older women's ( $>70$ years) perspective. Psychooncology 2008;17:410-416.

17. Cheville AL, Rhudy L, Basford JR, Griffin JM, Flores AM. How receptive are patients with late stage cancer to rehabilitation 
services and what are the sources of their resistance? Arch Phys Med Rehabil 2017;98:203-210.

18. Fu JB, Lee J, Smith DW, Shin K, Guo Y, Bruera E. Frequency and reasons for return to the primary acute care service among patients with lymphoma undergoing inpatient rehabilitation. PM R 2014;6:629-634.

19. Fu JB, Lee J, Smith DW, Guo Y, Bruera E. Return to primary service among bone marrow transplant rehabilitation inpatients: An index for predicting outcomes. Arch Phys Med Rehabil 2013;94: 356-361.

20. Sabers SR, Kokal JE, Girardi JC, et al. Evaluation of consultationbased rehabilitation for hospitalized cancer patients with functional impairment. Mayo Clin Proc 1999;74:855-861.

21. Montagnini M, Lodhi M, Born W. The utilization of physical therapy in a palliative care unit. J Palliat Med 2003;6:11-17.

22. Yoshioka H. Rehabilitation for the terminal cancer patient. Am J Phys Med Rehabil 1994;73:199-206.

23. Mackey KM, Sparling JW. Experiences of older women with cancer receiving hospice care: Significance for physical therapy. Phys Ther 2000;80:459-468.

24. Pyszora A, Budzynski J, Wojcik A, Prokop A, Krajnik M. Physiotherapy programme reduces fatigue in patients with advanced cancer receiving palliative care: Randomized controlled trial. Support Care Cancer 2017;25:2909.

25. Litterini AJ, Fieler VK, Cavanaugh JT, Lee JQ. Differential effects of cardiovascular and resistance exercise on functional mobility in individuals with advanced cancer: A randomized trial. Arch Phys Med Rehabil 2013;94:2329-2335.

26. Oldervoll LM, Loge JH, Lydersen S, et al. Physical exercise for cancer patients with advanced disease: A randomized controlled trial. Oncologist 2011;16:1649-1657.

27. Feldstain A, MacDonald N, Bhargava R, Chasen M. Reported distress in patients living with advanced cancer: Changes pre-post interdisciplinary palliative rehabilitation. Support Care Cancer 2017 May 4 [Epub ahead of print].

28. Oldervoll LM, Loge JH, Paltiel H, et al. The effect of a physical exercise program in palliative care: A phase II study. J Pain Symptom Manage 2006;31:421-430.

29. Chasen MR, Feldstain A, Gravelle D, Macdonald N, Pereira J. An interprofessional palliative care oncology rehabilitation program: Effects on function and predictors of program completion. Curr Oncol 2013;20:301-309.

30. Clark MM, Rummans TA, Atherton PJ, et al. Randomized controlled trial of maintaining quality of life during radiotherapy for advanced cancer. Cancer 2013;119:880-887.

31. Temel JS, Greer JA, Goldberg S, et al. A structured exercise program for patients with advanced non-small cell lung cancer. J Thorac Oncol 2009;4:595-601.

32. Quist M, Langer SW, Rorth M, Christensen KB, Adamsen L. "EXHALE": Exercise as a strategy for rehabilitation in advanced stage lung cancer patients: A randomized clinical trial comparing the effects of 12 weeks supervised exercise intervention versus usual care for advanced stage lung cancer patients. BMC Cancer 2013;13:477.

33. Jones L, Fitzgerald G, Leurent B, et al. Rehabilitation in advanced, progressive, recurrent cancer: A randomized controlled trial. J Pain Symptom Manage 2013;46:315-325.

34. Round J, Leurent B, Jones L. A cost-utility analysis of a rehabilitation service for people living with and beyond cancer. BMC Health Serv Res 2014;14:558.

35. Sarmento VP, Gysels M, Higginson IJ, Gomes B. Home palliative care works: but how? A meta-ethnography of the experiences of patients and family caregivers. BMJ Support Palliat Care 2017 Feb 23 [Epub ahead of print].

36. Gomes B, Calanzani N, Curiale V, McCrone P, Higginson IJ. Effectiveness and cost-effectiveness of home palliative care services for adults with advanced illness and their caregivers. Cochrane Database Syst Rev 2013:CD007760.
37. Savio C, Garaventa A, Gremmo M, et al. Feasibility of integrated home/hospital physiotherapeutic support for children with cancer. Support Care Cancer 2007;15:101-104.

38. Cheville AL, Kollasch J, Vandenberg J, et al. A home-based exercise program to improve function, fatigue, and sleep quality in patients with stage IV lung and colorectal cancer: A randomized controlled trial. J Pain Symptom Manage 2012;45:811-821.

39. Kroenke K, Theobald D, Wu J, et al. Effect of telecare management on pain and depression in patients with cancer: A randomized trial. JAMA 2010;304:163-171.

40. Johns SA, Kroenke K, Krebs EE, Theobald DE, Wu J, Tu W. Longitudinal comparison of three depression measures in adult cancer patients. J Pain Symptom Manage 2013;45:71-82.

41. Johns SA, Brown LF, Beck-Coon K, et al. Randomized controlled pilot trial of mindfulness-based stress reduction compared to psychoeducational support for persistently fatigued breast and colorectal cancer survivors. Support Care Cancer 2016;24: 4085-4096.

42. Johns SA, Brown LF, Beck-Coon K, Monahan PO, Tong Y, Kroenke K. Randomized controlled pilot study of mindfulness-based stress reduction for persistently fatigued cancer survivors. Psychooncology 2015;24:885-893.

43. Shepherd L, Goldstein D, Whitford H, Thewes B, Brummell V, Hicks $M$. The utility of videoconferencing to provide innovative delivery of psychological treatment for rural cancer patients: Results of a pilot study. J Pain Symptom Manage 2006;32: 453-461.

44. Cluver JS, Schuyler D, Frueh BC, Brescia F, Arana GW. Remote psychotherapy for terminally ill cancer patients. J Telemed Telecare 2005;11:157-159.

45. Mair F, Whitten P. Systematic review of studies of patient satisfaction with telemedicine. BMJ 2000;320:1517-1520.

46. Stubblefield MD. Commentary on "Oncologists' and physiatrists' attitudes regarding rehabilitation for patients with advanced cancer." PM R 2012;4:109-110.

47. Stout NL, Andrews K, Binkley JM, Schmitz KH, Smith RA. Stakeholder perspectives on dissemination and implementation of a prospective surveillance model of rehabilitation for breast cancer treatment. Cancer 2012;118:2331-2334.

48. Runacres F, Gregory H, Ugalde A. 'The horse has bolted I suspect': A qualitative study of clinicians' attitudes and perceptions regarding palliative rehabilitation. Palliat Med 2017;31: $642-650$

49. White DB, Ernecoff N, Buddadhumaruk P, et al. Prevalence of and factors related to discordance about prognosis between physicians and surrogate decision makers of critically ill patients. JAMA 2016; 315:2086-2094.

50. Gramling R, Fiscella K, Xing G, et al. Determinants of patientoncologist prognostic discordance in advanced cancer. JAMA Oncol 2016;2:1421-1426.

51. Hui D, Cerana MA, Park M, Hess K, Bruera E. Impact of oncologists' attitudes toward end-of-life care on patients' access to palliative care. Oncologist 2016;21:1149-1155.

52. Giesinger JM, Wintner LM, Oberguggenberger AS, et al. Quality of life trajectory in patients with advanced cancer during the last year of life. J Palliat Med 2011;14:904-912.

53. Cheville AL, Yost KJ, Larson DR, et al. Performance of an item response theory-based computer adaptive test in identifying functional decline. Arch Phys Med Rehabil 2012;93:1153-1160.

54. Abdel-Wahab N, Shah M, Suarez-Almazor ME. Adverse events associated with immune checkpoint blockade in patients with cancer: A systematic review of case reports. PLoS One 2016;11: e0160221.

55. Kroenke K, Spitzer RL, Williams JB. The PHQ-9: Validity of a brief depression severity measure. J Gen Intern Med 2001;16:606-613.

56. Spitzer RL, Kroenke K, Williams JB, Lowe B. A brief measure for assessing generalized anxiety disorder: The GAD-7. Arch Intern Med 2006;166:1092-1097. 
57. Shi Q, Mendoza TR, Dueck AC, et al. Determination of mild, moderate, and severe pain interference in patients with cancer. Pain 2017;158:1108-1112.

58. Chochinov HM, Cann B, Cullihall K, et al. Dignity therapy: A feasibility study of elders in long-term care. Palliat Support Care 2012;10:3-15.

59. Ostrem W. Presentation to the ABIM Hospice and Palliative Medicine Exam Writing Committee. 2017 March 8. Philadelphia, PA: American Board of Internal Medicine.
60. Davis MP, Walsh D. Epidemiology of cancer pain and factors influencing poor pain control. Am J Hosp Palliat Care 2004;21: 137-142.

61. Cheville AL, Andrews K, Kollasch J, Schmidt K, Basford J. Adapting lymphedema treatment to the palliative setting. Am J Hosp Palliat Care 2014;31:38-44.

62. Parker PA, Baile WF, de Moor C, Lenzi R, Kudelka AP, Cohen L. Breaking bad news about cancer: Patients' preferences for communication. J Clin Oncol 2001;19:2049-2056.

\section{Disclosure}

A.L.C. Department of Physical Medicine and Rehabilitation, Mayo Clinic, 200 First Street, SW, Rochester, MN 55905. Address correspondence to: A.L.C.; e-mail: cheville.andrea@mayo.edu

Disclosure: nothing to disclose

M.M. Department of Health Sciences Research, Mayo Clinic, Rochester, MN Disclosure: nothing to disclose

S.R.S. Department of Physical Medicine and Rehabilitation, University of Michigan, Ann Arbor, Ml

Disclosure: nothing to disclose
J.R.B. Department of Physical Medicine and Rehabilitation, Mayo Clinic, Rochester, $M N$

Disclosure: nothing to disclose

Submitted for publication May 5, 2017; accepted July 31, 2017. 